Yaacov Shavit

An Imaginary Trio 



\section{Yaacov Shavit}

\section{An Imaginary Trio}

King Solomon, Jesus, and Aristotle

\section{DE GRUYTER}


Die freie Verfügbarkeit der E-Book-Ausgabe dieser Publikation wurde ermöglicht durch den Fachinformationsdienst Jüdische Studien an der Universitätsbibliothek J. C. Senckenberg Frankfurt am Main und 18 wissenschaftliche Bibliotheken, die die Open-Access-Transformation in den Jüdischen Studien unterstützen.

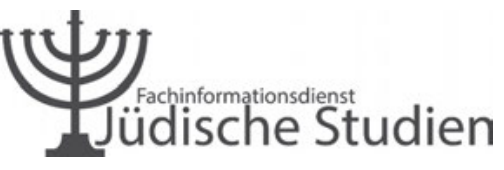

ISBN 978-3-11-067718-8

e-ISBN (PDF) 978-3-11-067726-3

e-ISBN (EPUB) 978-3-11-067730-0

\section{(cc) BY}

This work is licensed under the Creative Commons Attribution 4.0 International Licence. For details go to http://creativecommons.org/licenses/by/4.0/.

Das E-Book ist als Open-Access-Publikation verfügbar über www.degruyter.com, https://www.doabooks.org und https://www.oapen.org

Library of Congress Control Number: 2020909307

\section{Bibliographic information published by the Deutsche Nationalbibliothek}

The Deutsche Nationalbibliothek lists this publication in the Deutsche Nationalbibliografie; detailed bibliographic data are available on the Internet at http://dnb.dnb.de.

(C) 2020 Yaacov Shavit, published by Walter de Gruyter GmbH, Berlin/Boston Cover image: Statue of King Solomon and Christ in the center of the southern portal of the cathedral Notre-Dame of Strasbourg (Bas-Rhin, France), Wikimedia Commons, Public Domain. Printing and binding: $\mathrm{CPI}$ books $\mathrm{GmbH}$, Leck

www.degruyter.com 


\section{Open-Access-Transformation in den Jüdischen Studien}

Open Access für exzellente Publikationen aus den Jüdischen Studien: Dies ist das Ziel der gemeinsamen Initiative des Fachinformationsdiensts Jüdische Studien an der Universitätsbibliothek J. C. Senckenberg Frankfurt am Main und des Verlags Walter De Gruyter. Unterstützt von 18 Konsortialpartnern können 2020 insgesamt acht Neuerscheinungen im Open Access Goldstandard veröffentlicht werden, darunter auch diese Publikation.

Die nachfolgenden wissenschaftlichen Einrichtungen haben sich an der Finanzierung beteiligt und fördern damit die Open-Access-Transformation in den Jüdischen Studien und gewährleisten die freie Verfügbarkeit für alle:

Fachinformationsdienst Jüdische Studien, Universitätsbibliothek J. C. Senckenberg Frankfurt am Main

Staatsbibliothek zu Berlin - Preußischer Kulturbesitz

Universitätsbibliothek der Freien Universität Berlin

Universitätsbibliothek der Technischen Universität Berlin

Universitäts- und Landesbibliothek Düsseldorf

Universitätsbibliothek der Europa-Universität Viadrina Frankfurt (Oder)

Bibliothek der Vereinigten Theologischen Seminare der Georg-August-Universität Göttingen Niedersächsische Staats- und Universitätsbibliothek Göttingen

Universitäts- und Landesbibliothek Sachsen-Anhalt

Staats- und Universitätsbibliothek Hamburg - Carl von Ossietzky

Gottfried Wilhelm Leibniz Bibliothek - Niedersächsische Landesbibliothek

Hochschule für Jüdische Studien Heidelberg

Universitäts- und Stadtbibliothek Köln

Universitätsbibliothek Mainz

Universitätsbibliothek der Ludwig-Maximilians-Universität München

Universitäts- und Landesbibliothek Münster

Herzog August Bibliothek Wolfenbüttel

Universitätsbibliothek Wuppertal 
